\title{
Whole-exome sequencing reveals MYH7 p.R671C mutation in three different phenotypes of familial hypertrophic cardiomyopathy
}

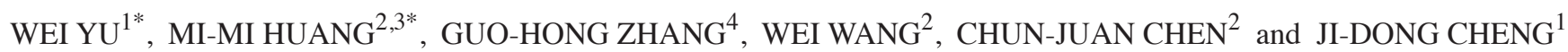 \\ ${ }^{1}$ Department of Internal Medicine, Xiang'an Hospital of Xiamen University, School of Medicine Xiamen University, Xiamen, \\ Fujian 361102; ${ }^{2}$ Department of Cardiology, The Second Affiliated Hospital of Shantou University Medical College, Shantou, \\ Guangdong 515041; ${ }^{3}$ Department of Internal Medicine, The First Affiliated Hospital of Guangxi Medical University, Nanning, \\ Guangxi 530021; ${ }^{4}$ Department of Pathology, Shantou University Medical College, Shantou, Guangdong 515041, P.R. China
}

Received July 30, 2020; Accepted January 6, 2021

DOI: $10.3892 /$ etm.2021.10434

\begin{abstract}
Familial hypertrophic cardiomyopathy (HCM) is one of the most common types of genetic heart disorder and features high genetic heterogeneity. HCM is a major cause of sudden cardiac death and also an important cause of heart failure-related disability. A pedigree with suspected familial HCM was recruited for the present study to identify genetic abnormalities. HCM was confirmed by echocardiography and clinical data of the family members were collected. Genomic DNA was extracted from the peripheral blood and sequenced based on standard whole-exome sequencing (WES) protocols. Sanger sequencing was further performed to verify mutation sites and their association with HCM. WES and Sanger sequencing revealed a heterozygous missense mutation (c.2011C > T p.R671C) in myosin heavy chain 7 (MYH7) that was identified in three family members. The Arg671Cys mutation was located in exon 18 and, to the best of our knowledge, has not been previously reported in familial HCM. Furthermore, family members carrying the same mutated gene were of different sexes and clinical phenotypes. They included the
\end{abstract}

Correspondence to: Dr Chun-Juan Chen, Department of Cardiology, The Second Affiliated Hospital of Shantou University Medical College, 69 Dong Xia North Road, Shantou, Guangdong 515041, P.R. China

E-mail: janey_stu@163.com

Professor Ji-Dong Cheng, Department of Internal Medicine, Xiang'an Hospital of Xiamen University, School of Medicine Xiamen University, 2000 Xiang'an East Road, Xiamen, Fujian 361102, P.R. China

E-mail: jidongcheng36@hotmail.com

${ }^{*}$ Contributed equally

Key words: familial hypertrophic cardiomyopathy, gene mutation, whole-exome sequencing, myosin heavy chain 7 , genotype-phenotype correlation proband, a 17-year-old survivor of sudden cardiac arrest with ventricular systolic dysfunction, the proband's maternal uncle, who presented with ventricular diastolic dysfunction and the proband's mother, who had no obvious clinical symptoms and did not present with cardiac dysfunction. However, echocardiology indicated that the proband's mother had an enlarged left atrium, slightly thicker right anterior wall and anterior septum and an expanded atrial septum. Therefore, HCM exhibited obvious genetic and phenotypic heterogeneity. To the best of our knowledge, the present study was the first to report such a mutation in the MYH7 gene in familial HCM. In addition, the present study demonstrated that WES is a powerful tool for identifying genetic variants in HCM.

\section{Introduction}

Hypertrophic cardiomyopathy (HCM) is a primary autosomal-dominant inherited myocardial disorder characterized by unexplained left ventricular hypertrophy (1). Studies over the last 50 years have indicated that HCM is a familial heart disease with a risk of sudden cardiac death (SCD). SCD, heart failure and thromboembolism are the three major causes of HCM-related death (2). SCD is mostly associated with fatal arrhythmias, which mainly include ventricular tachycardia (continuous or non-sustained), ventricular fibrillation and atrioventricular blockage. Furthermore, $\sim 1$ million individuals in China suffer from this disease, with an annual mortality rate of $3-6 \%$. HCM is one of the leading causes of sudden death in adolescents and athletes $(3,4)$.

HCM is the most common monogenic heart disease, and its causative genes are relatively well defined (5). It is mainly caused by a mutation in a gene that encodes a sarcomeric structural protein. The most common disease-causing genes encode myosin heavy chain 7 (MYH7), myosin binding protein C3 (MYBPC3), tropomyosin 1 (TPM1), troponin T2, cardiac type (TNNT2), TNNI3, actin alpha cardiac muscle 1 (ACTC1), myosin regulatory light chain 2 (MYL2) and MYL3 proteins (6-11). FH2 domain-containing protein 3 is a novel pathogenic gene of HCM (12). Among these genes, HCM caused by MYH7 gene mutations accounts for $30-50 \%$ 
of the total number of cases (13). Although the MYH7 gene is currently considered to be the major causative gene of HCM, the mechanisms by which its mutations cause HCM have remained to be fully elucidated. Currently recognized mechanisms include $\mathrm{Ca}^{2+}$ homeostasis, myocardial fibrosis and energy imbalance $(14,15)$. The present study performed whole-exome sequencing (WES) combined with Sanger sequencing to identify genetic abnormalities in a pedigree with familial HCM.

\section{Patients and methods}

Subjects. The subjects of the present study were a Han Chinese family with HCM from Guangdong (China). A pedigree chart is provided in Fig. 1. The proband (patient III-3) was a 17-year-old male (born in November 2000) who was admitted to the Second Affiliated Hospital of Shantou University Medical College (Shantou, China) with sudden cardiac arrest in September 2017. Based on the 2014 European Society of Cardiology (ESC) guidelines (16), the diagnostic criteria for HCM in adults are as follows: Arbitrary imaging (echocardiography, cardiac magnetic resonance imaging or computed tomography) indicated that a segment or segments of the left ventricular myocardium are not entirely due to abnormal cardiac load and segment wall thickness $\geq 15 \mathrm{~mm}$ (in adults) or left ventricular wall thickness $\geq$ predicted mean $+2 \mathrm{X}$ the standard deviation (in children aged $<14$ years). If for the first-degree relatives of the patient, after exclusion of other causes, cardiac imaging suggests that a segment of the left ventricular wall or a multi-stage thickness of $\geq 13 \mathrm{~mm}$ is present, a diagnosis of familial HCM may be made (16). According to the aforementioned information, the proband's uncle (patient II-2) met the diagnostic criteria for HCM but had no clinical symptoms. In addition, the proband's mother (patient II-3) also exhibited structural changes on echocardiography but no clinical symptoms. The proband's grandfather (I-1) suffered a sudden cardiac death at 40 years of age. The echocardiography result was normal in the grandmother (65-year-old) of the proband (I-2), who was set as the control in the present study. Hence, in the present study, four participants from three generations were investigated (I-2, II-2, II-3 and III-3; Fig. 1). Clinical data included results of physical examinations, laboratory tests (liver and kidney function, blood routine, rheumatoid immune index and sex hormone levels), electrocardiography and two-dimensional and Doppler echocardiographic examinations. All subjects signed informed consent forms. The present study was implemented strictly in accordance with the Declaration of Helsinki and approved by the Ethics Committee of the Second Affiliated Hospital of Shantou University Medical College (Shantou, China).

WES and mutation screening. To extract genomic DNA, peripheral venous blood was collected from the participants and EDTA was used as the anticoagulant. A blood extraction kit (cat. no. 51304; Invitrogen; Thermo Fisher Scientific, Inc.) was used to extract genomic DNA from leukocytes according to the manufacturer's protocol. Extracted DNA samples were sent to Beijing Nuohe Zhiyuan Technology Co., Ltd. for WES. Genomic DNA was sheared into fragments with a length of 150-200 bp through sonication (17). End-repairing, A-tailing and adaptor ligation, a four-cycle pre-capture PCR amplification and xGen Exome Research Panel (Integrated DNA Technologies, Inc.) enrichment were then performed (17). The sequencing was performed on an Illumina Hiseq Xten platform (Illumina, Inc.) with a mean sequence coverage of $\geq 90 \mathrm{X}$ and a coverage of $\geq 20 \mathrm{X}$ in $>95 \%$ of the target bases. Cutadapt (https://cutadapt.readthedocs.io/en/stable/) and FastQC (http://darlinglab.org/tutorials/fastqc/) were used to perform quality control. Clean reads were mapped to the human reference genome (University of California Santa Cruz hg19) via Sentieon BWA (version 0.7.15) (18). Duplicate sequence reads were removed using Picard (version 1.85) and variants were detected by GATK (version 3.1) (19). Variants were annotated using ANNOVAR software (version from 14 December 2015; http://annovar.openbioinformatics.org/en/latest/). All exome variants were filtered for allele frequencies $<0.001$ in the ExAC database (20). The nomenclature of variants was based on the guidelines of the Human Genome Variation Society (21).

Sanger sequencing. Sanger sequencing was performed to verify the variants in MYH7 identified by WES. PCR was performed to amplify the exon of MYH7. The upstream sequence of the primer was 5'-CATCTCCTGGCCTCTTCA CTTA-3' and the downstream sequence was 5'-ATGTCCATC AGAGTGCCTTACA-3'. PCR was done using SYBR Premix EX Taq ${ }^{\mathrm{TM}}$ II (Takara Bio, Inc.). The following thermocycling conditions were used for the PCR: Initial denaturation at $94^{\circ} \mathrm{C}$ for $3 \mathrm{~min} ; 33$ cycles of denaturation at $94^{\circ} \mathrm{C}$ for $30 \mathrm{sec}$, annealing at $57^{\circ} \mathrm{C}$ for $45 \mathrm{sec}$ and extension at $72^{\circ} \mathrm{C}$ for $45 \mathrm{sec}$, followed by a final extension at $72^{\circ} \mathrm{C}$ for $10 \mathrm{~min}$. PCR products were then examined for sequence variations by Sanger sequencing.

Bioinformatics analysis. The gene detection strategies adopted in the present study are presented in Fig. 2. The frequencies of mutations in the general population were confirmed using the ExAC_EAS database (https://exac.hms.harvard.edu), gnomAD database (version r2.0; http://gnomad.brodinstitute. org) and 1000 Genomes project (http://www.1000genomes. org). The pathogenicity of the mutations was predicted using the single nucleotide polymorphism database (dbSNP; http://www.ncbi.nlm.nih.gov/SNP/) and Polyphen-HCM (http://www.genetics.bwh.harvard.edu/hcm) and they were annotated according to the recommendations of the American College of Medical Genetics and Genomics (22).

\section{Results}

Clinical phenotype. The proband (III-3) was a 17-year-old male who was admitted to The Second Affiliated Hospital of Shantou University Medical College (Shantou, China) after successful cardio-pulmonary resuscitation (CPR) due to cardiac arrest in September 2017. The patient had no chest pain, no history of syncope and no known family history of hereditary diseases. The patient's grandfather had died of a heart attack at the age of 40 years, but it was not confirmed whether this was due to HCM. On admission, the proband had a grade III systolic murmur at the apex of the heart. Electrocardiographic examination indicated abnormal Q waves, ST segment depression, $\mathrm{T}$ wave inversion and third-degree atrioventricular block 


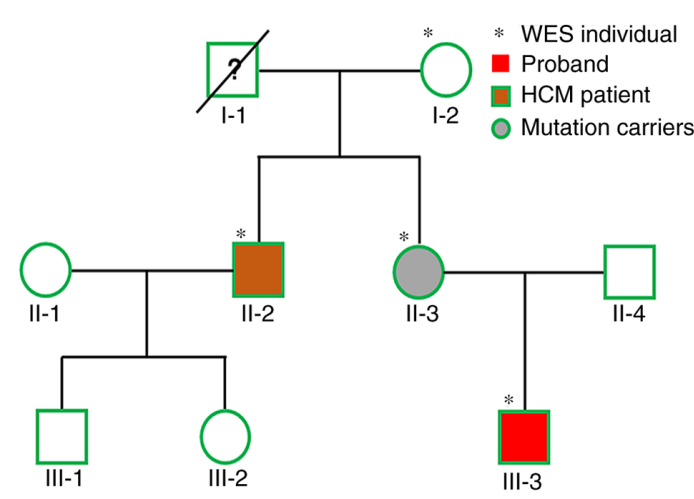

Figure 1. Family pedigree. III-3, the proband; II-2, patient with hypertrophic cardiomyopathy (the proband's uncle); II-3, carrier with mutation (the proband's mother); I-2, normal control (the proband's grandmother); I-1, death without definite disease (the proband's grandfather). WES, whole-exome sequencing; HCM, hypertrophic cardiomyopathy.

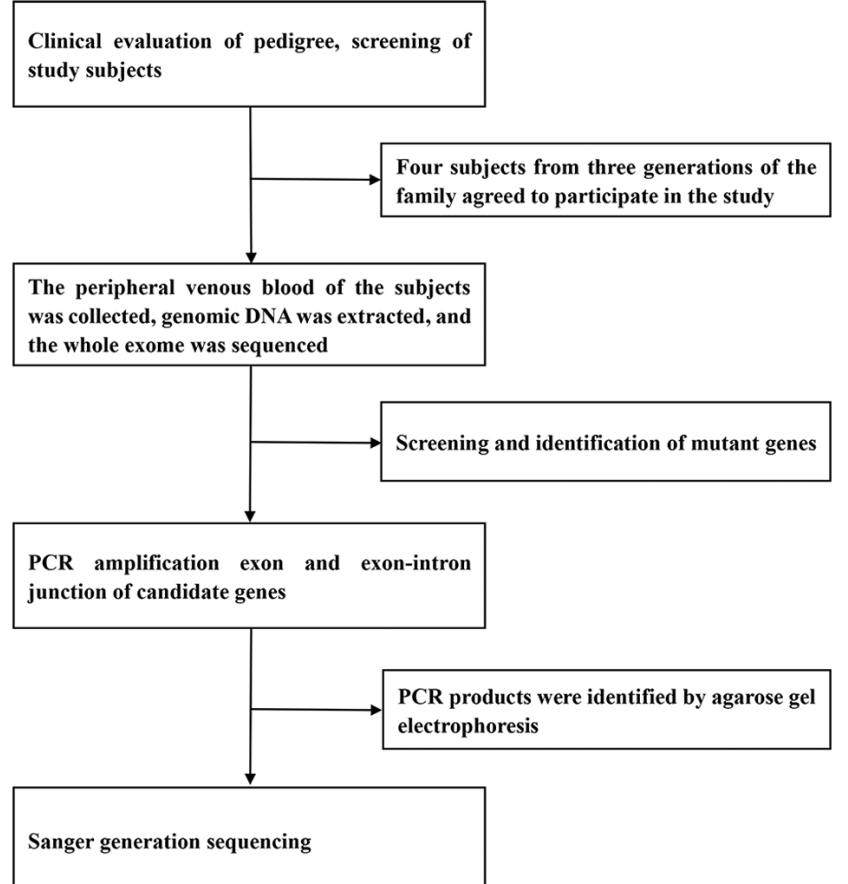

Figure 2. Flowchart of genetic testing strategy.

(Fig. 3A). Echocardiography displayed the ventricular septum (IVS; $15.3 \mathrm{~mm}$ ) and the posterior wall of the left ventricle (LVPW; $13.5 \mathrm{~mm}$; Table I; Fig. 3B). Examination of the thickness of the left ventricular short-axis papillary muscle suggested that the thickness of the hypertrophic myocardium was $20 \mathrm{~mm}$ (normal value, $<12 \mathrm{~mm}$ ), the anterior wall was $19 \mathrm{~mm}$ (normal value, $<12 \mathrm{~mm}$ ) and the lower wall was $19 \mathrm{~mm}$ (normal value, $<12 \mathrm{~mm}$ ). All of these data were above the diagnostic lower limit of $15 \mathrm{~mm}$ for HCM. Therefore, the proband was diagnosed with HCM according to the ESC criteria aforementioned (16).

Detailed clinical data of the family are presented in Table I and Fig. 3. The proband's uncle (II-2) was also confirmed to have HCM by echocardiography (IVS, $15.4 \mathrm{~mm}$; LVPW, $12.4 \mathrm{~mm}$ ) and presenting with cardiac dysfunction (Table I; Fig. 3D). Although this was not clinically confirmed according to the HCM diagnostic criteria, echocardiology indicated

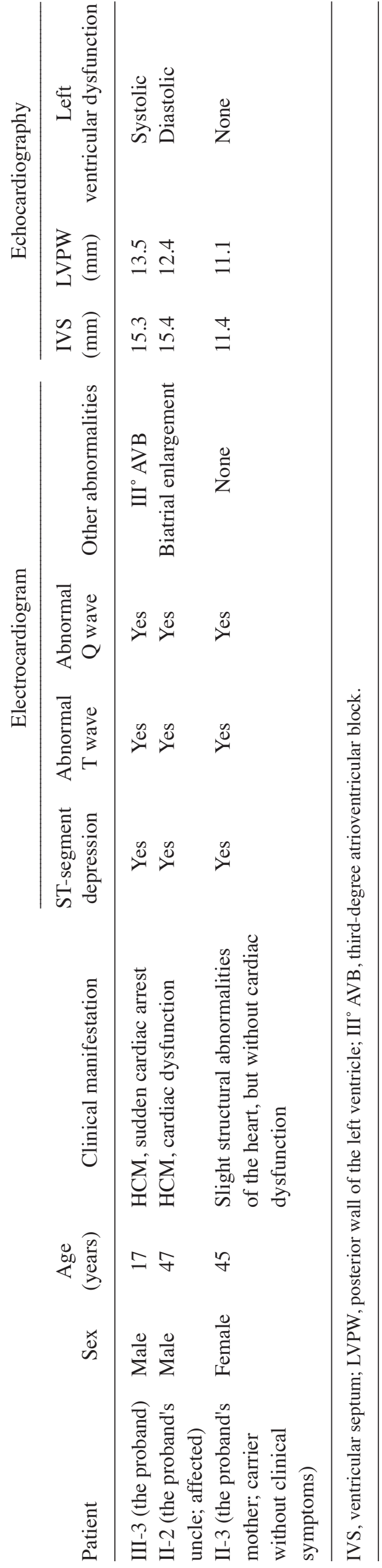



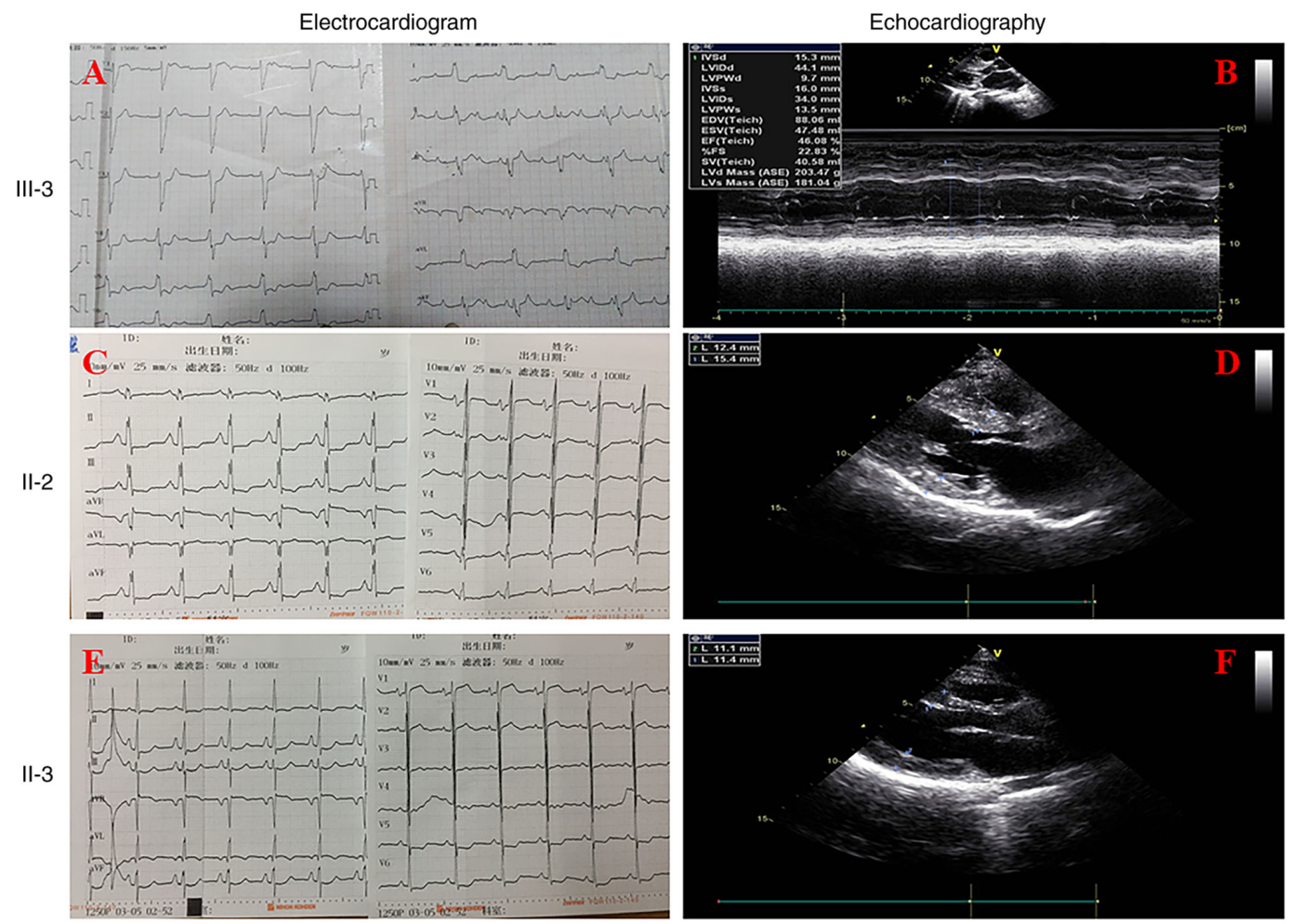

Figure 3. Electrocardiograms and echocardiographs of members of the pedigree. (A and B) III-3, the proband. (A) Electrocardiogram and (B) echocardiograph. (C and D) II-2, patient with hypertrophic cardiomyopathy (the proband's uncle). (C) Electrocardiogram and (D) echocardiograph. (E and F) II-3, carrier with mutation (the proband's mother). (E) Electrocardiogram and (F) echocardiograph.

that the proband's mother (II-3) had an enlarged left atrium, slightly thicker right anterior wall and anterior septum and an expanded atrial septum (Table I; Fig. 3F). The function of the heart was normal in the proband's grandmother. Laboratory tests (liver and kidney function, blood routine testing, rheumatoid immune index and sex hormone levels) of all subjects in the present study provided normal results. In addition, none of the subjects in this study had living habits that are considered to be risk factors, including smoking and drinking.

Genetic screening. DNA samples from four members (I-2, II-2, II-3 and III-3) of the family were subjected to WES analysis (Fig. 1). Previous studies have reported that mutations in 57 genes may cause or be associated with HCM, including eight definitive genes (MYBPC3, MYH7, TNNT2, TNNI3, TPM1, ACTC1, MYL2 and MYL3), three moderately evidenced genes [cysteine and glycine-rich protein 3 (CSRP3), troponin $\mathrm{C}$ and junctophilin-2] and other genes with limited or no evidence (titin, Krueppel-like factor 10, myopalladin, ankyrin repeat domain-containing protein 1 , myosin light chain kinase 2, myozenin-2, nexilin, vinculin, E3 ubiquitin-protein ligase TRIM63, ryanodine receptor 2, MYH6, obscurin, PDZ and LIM domain protein 3, telethonin, myomesin-1 and calreticulin-3) (23-27). Therefore, the present study focused on the genetic variations (SNPs and indels) occurring in any of the aforementioned genes. Furthermore, the present study considered only the genetic variations that existed in both affected patients. The left non-synonymous single nucleotide variants (SNVs) were prioritized by the SNV Prioritization via the Integration of Genomic data (SPRING) (http://bioinfo. au.tsinghua.edu.cn/spring).
Based on the aforementioned strategy for variation analysis, 21 variants were obtained in the present study (19 missense SNVs and two stopgain SNVs) in 21 genes, of which only the MYH7 mutation exhibited a close association with HCM (Table II). The mutation is located on chromosome 14 and the SNP site (ID: rs727503263) is a heterozygous missense mutation in the 18th exon. This mutation corresponds to a one-base substitution at 2011 (C to T), at position 671 of the amino acid sequence. The mutation resulted in an amino acid change (arginine to cysteine) with a frequency of 0 in gnom $A D$ and 1,000 Genomes and $<0.001$ in the ExAC_EAS exome or genome sequencing databases (Table III). The p.Arg671 variant is highly conserved in mammals, from humans to house mouse (Fig. 4). In silico analysis by Polyphen-HCM and SIFT suggested that the p.R671C variant is a disease-causing variant (Table III). This variant was also interpreted as being a likely pathogenic variant by ClinVar (https://www.ncbi.nlm. nih.gov/clinvar/variation/164350/). In order to confirm the mutation, the Sanger generation of the family members were sequenced and verified for the mutation. Family members with echocardiology changes (II-2, II-3 and III-3) harbored the same combination of MYH7 mutations, while the healthy member (I-2) exhibited no mutations at this position (Fig. 5). These results are consistent with the electrocardiograms, echocardiography and clinical symptoms.

Family genotype-phenotype analysis. After screening by exome sequencing, it was determined that three subjects in the family (II-2, II-3 and III-3), including the proband, carried a gene mutation of MYH7. However, these three subjects exhibited differences in their electrocardiograms, i.e. the 
Table II. SNVs with $\mathrm{P}<0.05$ analyzed by SNV Prioritization via the Integration of Genomic data.

\begin{tabular}{|c|c|c|c|c|c|c|c|}
\hline Gene & $\begin{array}{l}\text { Amino } \\
\text { acid change }\end{array}$ & $\begin{array}{c}\text { Type } \\
\text { of variant }\end{array}$ & dbSNP (rsID) & SIFT & PolyPhen2 & $\begin{array}{c}\text { Likelihood } \\
\text { ratio test }\end{array}$ & MutationTaster \\
\hline MYH7 & R671C & Missense & rs727503263 & $\mathrm{D}$ & $\mathrm{D}$ & $\mathrm{D}$ & $\mathrm{D}$ \\
\hline MIB2 & R385W & Missense & rs376615315 & $\mathrm{D}$ & $\mathrm{D}$ & $\mathrm{D}$ & $\mathrm{D}$ \\
\hline SIGLEC1 & $\mathrm{R} 400 \mathrm{C}$ & Missense & rs76254218 & $\mathrm{D}$ & $\mathrm{D}$ & $\mathrm{N}$ & $\mathrm{D}$ \\
\hline FNDC1 & L1839P & Missense & rs201022798 & $\mathrm{D}$ & $\mathrm{D}$ & $\mathrm{D}$ & $\mathrm{D}$ \\
\hline HTR1B & I368T & Missense & rs761871198 & $\mathrm{D}$ & $\mathrm{D}$ & $\mathrm{D}$ & $\mathrm{D}$ \\
\hline PCDHB10 & R289X & Stopgain & rs782513627 & Absent & Absent & Absent & $\mathrm{D}$ \\
\hline OR7A5 & M59V & Missense & rs756036560 & $\mathrm{D}$ & $\mathrm{D}$ & $\mathrm{U}$ & $\mathrm{N}$ \\
\hline DNAH14 & R2911C & Missense & rs770222940 & $\mathrm{D}$ & $\mathrm{D}$ & $\mathrm{N}$ & $\mathrm{N}$ \\
\hline YEATS2 & T739A & Missense & rs201274382 & $\mathrm{D}$ & $\mathrm{D}$ & D & $\mathrm{D}$ \\
\hline TRAPPC4 & D160H & Missense & Absent & $\mathrm{D}$ & $\mathrm{D}$ & $\mathrm{D}$ & $\mathrm{D}$ \\
\hline DONSON & P328L & Missense & Absent & $\mathrm{D}$ & $\mathrm{D}$ & $\mathrm{D}$ & $\mathrm{D}$ \\
\hline DBX2 & G11S & Missense & rs746897983 & $\mathrm{D}$ & $\mathrm{D}$ & $\mathrm{U}$ & $\mathrm{N}$ \\
\hline RIMKLA & P326Q & Missense & rs199674761 & $\mathrm{D}$ & $\mathrm{D}$ & $\mathrm{D}$ & $\mathrm{D}$ \\
\hline ALDH1L1 & D741H & Missense & rs746372949 & $\mathrm{D}$ & $\mathrm{D}$ & $\mathrm{D}$ & $\mathrm{D}$ \\
\hline SEMA3G & D64N & Missense & rs775338504 & $\mathrm{D}$ & $\mathrm{D}$ & $\mathrm{D}$ & $\mathrm{D}$ \\
\hline TMEM108 & S293C & Missense & Absent & $\mathrm{D}$ & $\mathrm{D}$ & $\mathrm{N}$ & $\mathrm{N}$ \\
\hline WRAP53 & $\mathrm{R} 250 \mathrm{P}$ & Missense & rs780547823 & $\mathrm{D}$ & $\mathrm{D}$ & $\mathrm{D}$ & $\mathrm{D}$ \\
\hline SGCA & R110Q & Missense & rs145697858 & $\mathrm{D}$ & $\mathrm{D}$ & $\mathrm{D}$ & $\mathrm{D}$ \\
\hline EXOSC9 & K67N & Missense & Absent & $\mathrm{D}$ & $\mathrm{D}$ & $\mathrm{D}$ & $\mathrm{D}$ \\
\hline DNAH7 & $\mathrm{R} 2105 \mathrm{X}$ & Stopgain & rs186849698 & Absent & Absent & $\mathrm{D}$ & Absent \\
\hline THAP6 & F207S & Missense & rs180792819 & $\mathrm{D}$ & $\mathrm{D}$ & Absent & $\mathrm{D}$ \\
\hline
\end{tabular}

SNP/V, single nucleotide polymorphism/variant; db, database; SIFT, Sorting Intolerant From Tolerant; D, deleterious; N, neutral; U, uncertain. ALDH1L1, aldehyde dehydrogenase 1 family member L1; DBX2, developing brain homeobox 2; DNAH14, dynein axonemal heavy chain 14; DNAH7, dynein axonemal heavy chain 7; DONSON, downstream neighbor of SON; EXOSC9, exosome component 9; FNDC1, fibronectin type III domain containing 1; HTR1B, 5-hydroxytryptamine receptor 1B; MIB2, mindbomb E3 ubiquitin protein ligase 2; MYH7, myosin heavy chain 7; OR7A5, olfactory receptor family 7 subfamily A member 5; PCDHB10, protocadherin beta 10; RIMKLA, ribosomal modification protein rimK-like family member A; SEMA3G, sema domain immunoglobulin domain short basic domain secreted 3G; TMEM108, transmembrane protein 108; SGCA, sarcoglycan alpha; SIGLEC1, sialic acid binding Ig-like lectin 1; THAP6, THAP domain containing 6; TRAPPC4; trafficking protein particle complex 4; WRAP53, WD repeat containing antisense to TP53; YEATS2; YEATS domain containing 2.

Table III. In silico analysis of MYH7 variants.

\begin{tabular}{|c|c|c|c|c|c|c|}
\hline Variant & Amino acid change & Polyphen-HCM ${ }^{\mathrm{a}}$ & $\mathrm{SIFT}^{\mathrm{b}}$ & ExAC_EAS ${ }^{c}$ & gnomAD $^{\mathrm{d}}$ & 1000 Genomes $^{\mathrm{e}}$ \\
\hline c. $2011 \mathrm{C}>\mathrm{T}$ & p.R671C & Probable damage (1.0) & Damaging (0) & $<0.001$ & 0 & 0 \\
\hline
\end{tabular}

degree of cardiac hypertrophy was not similar. The proband and the proband's uncle were diagnosed with HCM, but the proband's mother was not. In addition, the proband is a survivor of sudden cardiac arrest, while the proband's uncle presented with cardiac dysfunction. The proband's mother had no obvious clinical symptoms and was without cardiac dysfunction. All three family members had the same mutated gene, but had three different clinical phenotypes, which was particularly exhibited in the degree of cardiac hypertrophy and severity of clinical symptoms. To improve the prognosis, the family members were recommended to undergo clinical evaluations every 12-24 months, including $24 \mathrm{~h}$ ambulatory electrocardiogram and echocardiography.

\section{Discussion}

The present study was the first, to the best of our knowledge, to reveal a MYH7 p.R671C mutation in three members of the 


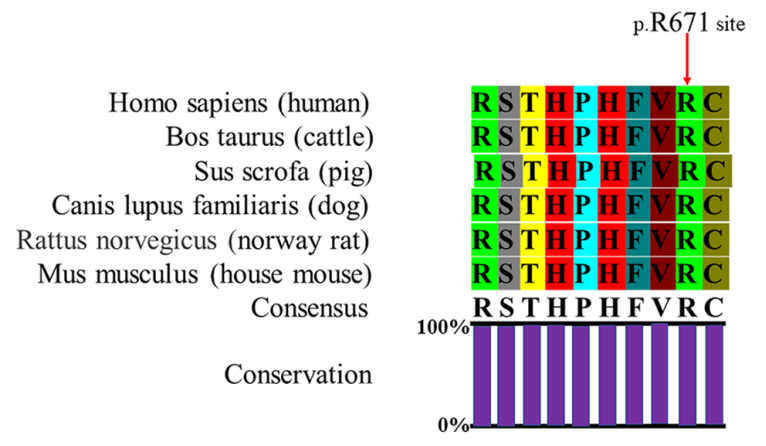

Figure 4. Sequence alignment of MYH7 protein in mammals. The red arrow indicates the Pro671 site, which is $100 \%$ conserved in mammals, from humans to house mouse. MYH7, myosin heavy chain 7.

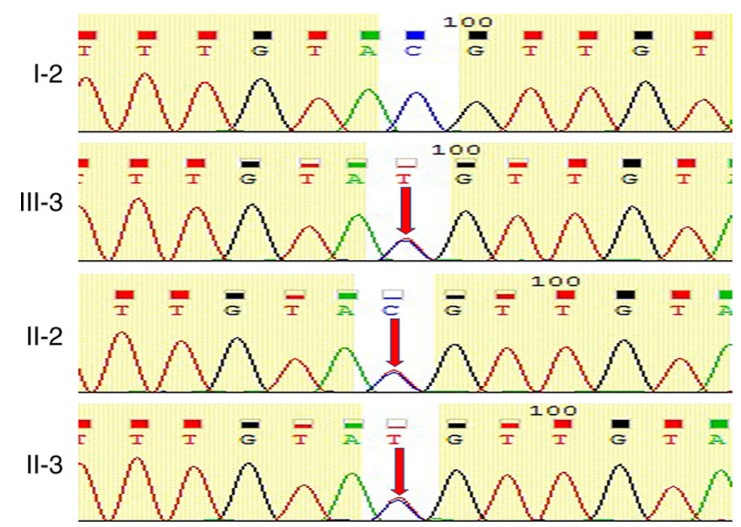

Figure 5. Sanger sequencing validating the MYH7 variant in the family. Sanger sequencing confirmed the heterozygous MYH7 variant in the family members. The red arrow points to the mutational site (c.2011C $>$ T p.R671C). I-2, normal control (the proband's grandmother); III-3, the proband; II-2, patient with hypertrophic cardiomyopathy (the proband's uncle); II-3, carrier with mutation (the proband's mother). MYH7, myosin heavy chain 7 .

same family with HCM based on WES combined with Sanger sequencing. Previous studies have confirmed that HCM is a single-gene disease with an autosomal-dominant inheritance pattern and nearly $60 \%$ of patients have familial aggregation $(28,29)$. The discovery of the p.Arg403Glu mutation in the MYH7 gene encoding the $\beta$-myosin heavy chain 7 protein in a French-Canadian family described by Perryman et al (30) was the foundation of subsequent important discoveries. Multiple independent mutations were identified from all genes encoding sarcomeric proteins and HCM is currently considered a genetically heterogeneous disease. Among the pathogenic genes known at this stage, MYH7 and MYBPC3 are the two most common and approximately half of HCM cases were caused by these two genes (7,31-33). Mutations in the TNNT2, TNNI3 and TPM1 genes, which account for HCM, are relatively rare and $\sim 10 \%$ of cases are caused by the aforementioned genes (33). Mutant genes such as ACTC1, MYL2, MYL3 and CSRP3 were also detected, these occur more rarely in patients with $\operatorname{HCM}$ (13). So far, although multitudinous disease-causing genes aforementioned have been found, their causal effects have remained to be fully elucidated. In the present study, an affected pedigree was subjected to gene screening through WES, which differs from multi-gene targeted sequencing used in most previous studies. The results suggested that the family carried a mutation in the MYH7 gene. This mutation corresponds to a one-base substitution at base 2,011 (C to T), resulting in an amino acid change at position 671 (arginine to cysteine). Although the mutation of MYH7 p.R671C has been previously reported in HCM, it was only in sporadic single cases $(10,34)$. To the best of our knowledge, the present study was the first to identify the mutation in three members of the same family with HCM.

In the present study, the family members carrying the same gene mutation displayed differences in sex and clinical phenotype. The proband and the proband's uncle were diagnosed with HCM, and while the proband's mother was classified as a mutation carrier. Although the proband's mother had some structural abnormalities in her heart, they did not meet the diagnostic criteria for HCM. The proband was a 17-year-old survivor of sudden cardiac arrest with systolic dysfunction. The proband's uncle presented with diastolic dysfunction. However, his mother has no obvious clinical symptoms, and without cardiac dysfunction. WES revealed that the three members of the family carried the mutant gene MYH7, which was the likely pathogenic mutation. The functional significance of this mutation, however, is not well understood in familial HCM. Among the pathogenic genes known at this stage, MYH7 and MYBPC 3 are the two most common, and about half of all HCM cases are caused by these two genes. Studies have reported that MYH7 mutation at different sites may result in different prognoses of patients, which also includes the influence of environmental differences, such as smoking and alcohol abuse. In view of phenotypic heterogeneity and gene polymorphisms in the family examined in the present study, an HCM model of MYH7 p.R671c mutation may be established by inducing pluripotent stem cells and cardiomyocytes to further study the potential molecular mechanism of cardiac hypertrophy (35).

In the present study, the results of WES and Sanger generation sequencing indicated that the proband, the proband's uncle and the proband's mother all carried a heterozygous mutation in the MYH7 gene. In addition, members of the family with the same mutation had different phenotypes. The degree of cardiac hypertrophy and cardiac dysfunction were different among family members of different sexes and age, reflecting genetic polymorphisms and phenotypic heterogeneity in HCM.

In conclusion, the present study first reported the p.Arg671Cys mutation of MYH7 as a likely pathogenic variant in familial HCM. In addition, the family members carrying the same mutated gene exhibited three distinct clinical phenotypes. Furthermore, the present study demonstrated that WES is a powerful tool for identifying genetic variants in HCM and may contribute to precise diagnosis and treatment.

\section{Acknowledgements}

Not applicable.

\section{Funding}

The study was supported by grants from the Medical Scientific Research Foundation of Guangdong Province (grant nos. A2019130 and A2019568). 


\section{Availability of data and materials}

The sequencing data used and/or analyzed during the current study are not deposited in a public database to protect patient privacy but are available from the corresponding author on reasonable request.

\section{Authors' contributions}

WY, MMH, CJC and JDC were involved in the analysis of the WES data. WY, MMH, GHZ and WW were involved in clinical data collection, performed data analysis and interpreted the results. CJC and JDC checked and confirmed the authenticity of the raw data. WY, CJC and JDC were involved in manuscript preparation. All authors read and approved the final manuscript.

\section{Ethics approval and consent to participate}

All experiments were approved by the Ethics Committee of the Second Affiliated Hospital of Shantou University Medical College (Shantou, China).

\section{Patient consent for publication}

Written informed consents were obtained from all the patients for the publication of all accompanying images.

\section{Competing interests}

The authors declare that they have no competing interests.

\section{References}

1. Maron BJ, Ommen SR, Semsarian C, Spirito P, Olivotto I and Maron MS: Hypertrophic cardiomyopathy: Present and future, with translation into contemporary cardiovascular medicine. J Am Coll Cardiol 64: 83-99, 2014.

2. Ho CY, Day SM, Ashley EA, Michels M, Pereira AC, Jacoby D, Cirino AL, Fox JC, Lakdawala NK, Ware JS, et al Genotype and lifetime burden of disease in hypertrophic cardiomyopathy: Insights from the sarcomeric human cardiomyopathy registry (SHaRe). Circulation 138: 1387-1398, 2018.

3. Spirito P and Autore C: Management of hypertrophic cardiomyopathy. BMJ 332: 1251-1255, 2006.

4. Maron BJ: Clinical course and management of hypertrophic cardiomyopathy. N Engl J Med 379: 655-668, 2018.

5. Coppini R, Santini L, Olivotto I, Ackerman MJ and Cerbai E: Abnormalities in sodium current and calcium homoeostasis as drivers of arrhythmogenesis in hypertrophic cardiomyopathy. Cardiovasc Res 116: 1585-1599, 2020.

6. Jääskeläinen P, Vangipurapu J, Raivo J, Kuulasmaa T, Heliö T, Aalto-Setälä K, Kaartinen M, Ilveskoski E, Vanninen S, Hämäläinen L, et al: Genetic basis and outcome in a nationwide study of Finnish patients with hypertrophic cardiomyopathy. ESC Heart Fail 6: 436-445, 2019.

7. Seeger T, Shrestha R, Lam CK, Chen C, McKeithan WL, Lau E, Wnorowski A, McMullen G, Greenhaw M, Lee J, et al: A premature termination codon mutation in MYBPC3 causes hypertrophic cardiomyopathy via chronic activation of nonsense-mediated decay. Circulation 139: 799-811, 2019.

8. Lopes LR, Zekavati A, Syrris P, Hubank M, Giambartolomei C, Dalageorgou C, Jenkins S, McKenna W; Uk10k Consortium, Plagnol V and Elliott PM: Genetic complexity in hypertrophic cardiomyopathy revealed by high-throughput sequencing. J Med Genet 50: 228-239, 2013.

9. Maron BJ, Maron MS and Semsarian C: Genetics of hypertrophic cardiomyopathy after 20 years: Clinical perspectives. J Am Coll Cardiol 60: 705-715, 2012.
10. Richard P, Charron P, Carrier L, Ledeuil C, Cheav T, Pichereau C, Benaiche A, Isnard R, Dubourg O, Burban M, et al: Hypertrophic cardiomyopathy: distribution of disease genes, spectrum of mutations, and implications for a molecular diagnosis strategy. Circulation 107: 2227-2232, 2003.

11. Toepfer CN, Wakimoto H, Garfinkel AC, McDonough B Liao D, Jiang J, Tai AC, Gorham JM, Lunde IG, Lun M, et al: Hypertrophic cardiomyopathy mutations in MYBPC3 dysregulate myosin. Sci Transl Med 11: eaat1199, 2019.

12. Ochoa JP, Sabater-Molina M, Garcia-Pinilla JM, Mogensen J, Restrepo-Córdoba A, Palomino-Doza J, Villacorta E, Martinez-Moreno M, Ramos-Maqueda J, Zorio E, et al: Formin homology 2 domain containing 3 (FHOD3) is a genetic basis for hypertrophic cardiomyopathy. J Am Coll Cardiol 72: 2457-2467, 2018.

13. Kaski JP, Syrris P, Esteban MT, Jenkins S, Pantazis A, Deanfield JE, McKenna WJ and Elliott PM: Prevalence of sarcomere protein gene mutations in preadolescent children with hypertrophic cardiomyopathy. Circ Cardiovasc Genet 2: 436-441, 2009.

14. Frey N, Luedde M and Katus HA: Mechanisms of disease: Hypertrophic cardiomyopathy. Nat Rev Cardiol 9: 91-100, 2011.

15. Sequeira V, Bertero E and Maack C: Energetic drain driving hypertrophic cardiomyopathy. FEBS Lett 593: 1616-1626, 2019.

16. Authors/Task Force members, Elliott PM, Anastasakis A, Borger MA, Borggrefe M, Cecchi F, Charron P, Hagege AA, Lafont A, Limongelli G, et al: 2014 ESC guidelines on diagnosis and management of hypertrophic cardiomyopathy: The task force for the diagnosis and management of hypertrophic cardiomyopathy of the European society of cardiology (ESC). Eur Heart J 35: 2733-2779, 2014.

17. Guo D, Shi Y, Jian W, Fu Y, Yang H, Guo M, Yong W, Chen G, Deng H, Qin Y, et al: A novel nonsense mutation in the L1CAM gene responsible for X-linked congenital hydrocephalus. J Gene Med 22: e3180, 2020.

18. Li H and Durbin R: Fast and accurate long-read alignment with burrows-wheeler transform. Bioinformatics 26: 589-595, 2010.

19. McKenna A, Hanna M, Banks E, Sivachenko A, Cibulskis K, Kernytsky A, Garimella K, Altshuler D, Gabriel S, Daly M and DePristo MA: The genome analysis toolkit: A MapReduce framework for analyzing next-generation DNA sequencing data. Genome Res 20: 1297-1303,2010.

20. Lek M, Karczewski KJ, Minikel EV, Samocha KE, Banks E, Fennell T, O'Donnell-Luria AH, Ware JS, Hill AJ, Cummings BB, et al: Analysis of protein-coding genetic variation in 60,706 humans. Nature 536: 285-291, 2016.

21. den Dunnen JT, Dalgleish R, Maglott DR, Hart RK, Greenblatt MS, McGowan-Jordan J, Roux AF, Smith T, Antonarakis SE and Taschner PE: HGVS recommendations for the description of sequence variants: 2016 update. Hum Mutat 37: 564-569, 2016.

22. Richards S, Aziz N, Bale S, Bick D, Das S, Gastier-Foster J, Grody WW, Hegde M, Lyon E, Spector E, et al: Standards and guidelines for the interpretation of sequence variants: A joint consensus recommendation of the American college of medical genetics and genomics and the association for molecular pathology. Genet Med 17: 405-424, 2015.

23. Ren MB, Chai XR, Li L, Wang X and Yin C: Potential digenic inheritance of familial hypertrophic cardiomyopathy identified by whole-exome sequencing. Mol Genet Genomic Med 8: e1150, 2020.

24. Das K J, Ingles J, Bagnall RD and Semsarian C: Determining pathogenicity of genetic variants in hypertrophic cardiomyopathy: Importance of periodic reassessment. Genet Med 16: 286-293, 2014

25. Green RC, Berg JS, Grody WW, Kalia SS, Korf BR, Martin CL, McGuire AL, Nussbaum RL, O'Daniel JM, Ormond KE, et al: ACMG recommendations for reporting of incidental findings in clinical exome and genome sequencing. Genet Med 15: 565-574, 2013.

26. Liew AC, Vassiliou VS, Cooper R and Raphael CE: Hypertrophic cardiomyopathy-past, present and future. J Clin Med 6: 118, 2017.

27. Ingles J, Goldstein J, Thaxton C, Caleshu C, Corty EW, Crowley SB, Dougherty K, Harrison SM, McGlaughon J, Milko LV, et al: Evaluating the clinical validity of hypertrophic cardiomyopathy genes. Circ Genom Precis Med 12: e002460, 2019. 
28. Valdés-Mas R, Gutiérrez-Fernández A, Gómez J, Coto E, Astudillo A, Puente DA, Reguero JR, Álvarez V, Morís C, León D, et al: Mutations in filamin C cause a new form of familial hypertrophic cardiomyopathy. Nat Commun 5: 5326, 2014.

29. Digilio MC, Pacileo G, Sarkozy A, Limongelli G, Conti E, Cerrato F, Marino B, Pizzuti A, Calabrò R and Dallapiccola B: Familial aggregation of genetically heterogeneous hypertrophic cardiomyopathy: A boy with LEOPARD syndrome due to PTPN11 mutation and his nonsyndromic father lacking PTPN11 mutations. Birth Defects Res A Clin Mol Teratol 70: 95-98, 2004.

30. Perryman MB, Yu QT, Marian AJ, Mares A Jr, Czernuszewicz G, Ifegwu J, Hill R and Roberts R: Expression of a missense mutation in the messenger RNA for beta-myosin heavy chain in myocardial tissue in hypertrophic cardiomyopathy. J Clin Invest 90: 271-277, 1992.

31. Lafreniere-Roula M, Bolkier Y, Zahavich L, Mathew J, George K, Wilson J, Stephenson EA, Benson LN, Manlhiot C and Mital S: Family screening for hypertrophic cardiomyopathy: Is it time to change practice guidelines? Eur Heart J 40 3672-3681, 2019.

32. Lopes LR, Brito D, Belo A and Cardim N; Portuguese Registry of Hypertrophic Cardiomyopathy: Genetic characterization and genotype-phenotype associations in a large cohort of patients with hypertrophic cardiomyopathy-an ancillary study of the Portuguese registry of hypertrophic cardiomyopathy. Int J Cardiol 278: 173-179, 2019
33. Millat G, Bouvagnet P, Chevalier P, Dauphin C, Jouk PS, Da Costa A, Prieur F, Bresson JL, Faivre L, Eicher JC, et al: Prevalence and spectrum of mutations in a cohort of 192 unrelated patients with hypertrophic cardiomyopathy. Eur J Med Genet 53: 261-267, 2010.

34. Mohiddin SA, Begley DA, McLam E, Cardoso JP, Winkler JB, Sellers JR and Fananapazir L: Utility of genetic screening in hypertrophic cardiomyopathy: Prevalence and significance of novel and double (homozygous and heterozygous) beta-myosin mutations. Genet Test 7: 21-27, 2003.

35. Jaffré F, Miller CL, Schänzer A, Evans T, Roberts AE, Hahn A and Kontaridis MI: Inducible pluripotent stem cell-derived cardiomyocytes reveal aberrant extracellular regulated kinase 5 and mitogen-activated protein kinase kinase $1 / 2$ signaling concomitantly promote hypertrophic cardiomyopathy in RAF1-associated noonan syndrome. Circulation 140: 207-224, 2019.

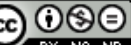

This work is licensed under a Creative Commons Attribution-NonCommercial-NoDerivatives 4.0 International (CC BY-NC-ND 4.0) License. 